\title{
Ginger relieves intestinal hypersensitivity of diarrhea predominant irritable bowel syndrome by inhibiting proinflammatory reaction
}

Changrong Zhang ${ }^{1+}$, Yongquan Huang ${ }^{2+}$, Peiwu $\mathrm{Li}^{3}$, Xinlin Chen ${ }^{4}$, Fengbin Liu ${ }^{3^{*}}$ and Qiuke Hou ${ }^{3,5^{*}}$ (I)

\begin{abstract}
Background: Ginger or ginger extracts have been used in traditional medicine relieve pain caused by diarrhea predominant irritable bowel syndrome (IBS-D), but few data exists about its effectiveness. This present study was to validate the effect of ginger on visceral pain, and to further explore the possible underlying mechanism by which ginger is used to relieve IBS-D intestinal hypersensitivity.

Methods: First, the IBS-D rat model was established by chemical stimulation and acute and chronic pressure stimulation. Then, different dose of ginger were administrated to IBS-D rats and evaluate the defecation frequency, fecal water content (FWC) and abdominal withdrawal reflex (AWR) scores in IBS-D rats. Further, the IBS-D rats were sacrificed to collecte the colonic tissues to evaluate the effect of ginger administration on its pathology and changes of pro-inflammatory factors, and changes of NF-KB pathway. Second, the ginger was taken to HPLC analysis and 6-gingerol was choosen to further experiment. Then, IBS-D rats were treated with different dose of 6gingerol, and the behavioral evaluation were to evaluate the effect of 6-gingerol on IBS-D rats. Further, colonic epithelial cells (CECS) were collectted and to evaluate the effect of 6-gingerol on the expression of inflammatory factors and changes of NF-KB pathway.
\end{abstract}

Results: The IBS-D rat model was successfully established by chemical stimulation and acute and chronic pressure stimulation. And ginger treatment significantly reduced the defecation frequency, fecal water content and AWR scores in IBS-D rats. Histopathological analysis showed that ginger treatment can significantly reduce colonic edema and promote the recovery of inflammation in IBS-D rats, and the effect is equivalent to rifaximin. Elisa and RT-qPCR showed that ginger inhibited the expression of proinflammatory factors (TNF-a, IL-6, iNOS) in IBS-D rats. Western blot showed IkBa was up-regulated while p-p65 was inhibited under ginger treatment. HPLC analysis showed that 6-gingerol was the main component of ginger, which could improve clinical symptoms in IBS-D rats. Western blot and RT-qPCR showed that 6-gingerol inhibited the expression of proinflammatory factors (TNF-a, IL-6, iNOS) in CECs, and inhibition of IKBa degradation and phosphorylation of p65 involved in NF-kB pathway.

(Continued on next page)

\footnotetext{
*Correspondence: liufb163@126.com; houqiuke@gzucm.edu.cn

${ }^{+}$Changrong Zhang and Yongquan Huang contributed equally to this work. ${ }^{3}$ Department of Gastroenterology, The First Affiliated Hospital of Guangzhou University of Chinese Medicine, Guangzhou, Guangdong 510405, P.R. China Full list of author information is available at the end of the article
} 
(Continued from previous page)

Conclusion: Ginger and ginger extract could relieve intestinal hypersensitivity of IBS-D by inhibiting proinflammatory response.

Keywords: Diarrhea predominant irritable bowel syndrome, Ginger, 6-gingerol, Intestinal hypersensitivity, NF-kB pathway

\section{Background}

Irritable bowel syndrome (IBS) is a chronic gastrointestinal disorder characterized by recurrent abdominal pain related to a change in bowel habit or defecation frenquency [1]. Diarrhea-predominant irritable bowel syndrome (IBS-D) is generally reported as the most common subtype (28-46\% of all IBS) [2]. IBS-D patients have reduced quality of life and may have poorer mood when in pain. Although most of the symptoms of IBS-D will disappear without the use of drugs, patients with severe abdominal pain need to consult a physician for medical treatment [3].

The therapeutic effect of IBS-D is limited, about 40\% of patients use alternative drugs to treat abdominal pain, bloating and other symptoms [4]. A randomized, double-blind, placebo-controlled trial have shown that rifaximin treatment is effective and well tolerated in IBSD, which could relieve IBS-D symptoms, including bloating, abdominal pain, and loose or watery stools [5]. However, the failure rate to relieve IBS-D abdominal pain by rifaximin treatment is between 20 and 25\% [6]. The most popular alternative medicine is ginger in a large study of 1012 patients with IBS-D [7]. Ginger, a traditional Chinese medicine, has been widely used for over 2500 years as an anti-inflammatory agent in musculoskeletal disorders [8]. Ginger is generally regarded as safe food by the US Food and Drug Administration, and can be exempted from pre-market review, approval and pre-market clinical testing (http://www.fda.gov/Food/ IngredientsPackagingLabeling/GRAS/ucm2006850.htm). There is no records of severe side effects or drug interactions in the Germany's Commission E Monograph [9]. Ginger root is the rhizome of the Zingiber officinale Roscoe, which usually contains $1-3 \%$ oil. The dosage of ginger is usually standardized according to the curcumin content, which is believed to have antibacterial effects [10], analgesics [11], antiemetics [12] and other physiological effects. In Micromedex (an evidence-based clinical reference tool for hospitals and physicians; www. micromedex.com) ginger is classified as a broadspectrum antiemetic drug, which can effectively treat nausea and pregnancy-related vomiting and surgery [13, 14]. Studies have found that ginger can affect pain and bowel movements, which suggests that ginger may help reduce pain and stool changes in IBS-D $[15,16]$. Given the known gastrointestinal effects of ginger and its widespread use and low cost, it should be tested as a potential treatment in patients with IBS.

Further, the severity of IBS-D with abdominal pain is directly proportional to the amounts of prostaglandin E2 (PGE2) released [17, 18]. A key step involving in PGE2 production is the conversion of arachidonic acid to prostanoids catalyzed by cyclooxygenase- 1 and -2 (COX-1, COX-2) [19]. Previous study have shown that 6-gingerol can inhibit the production of PGE2 and inflammation [20].

Though ginger is the most commonly used herbal medicines for IBS, there is few data exists about its effectiveness and underlying mechanisms. In order to clarify the mechanism of ginger alleviating abdominal pain in patients with IBS-D, we investigated the effect of ginger on an IBS-D rats model and IBS-D rats colonic epithelial cells. To explore the role of ginger in relievesrelieving intestinal hypersensitivity of IBS-D rats and improve clinical symptoms by prevent proinflammatory.

\section{Methods \\ Reagents and antibodies}

Acetic acid were purchased from Beijing Chemical Reagent Company (Beijing, China). Rifaximin (H20040042) was purchased from Sichuan Star Pharmaceutical Co., Ltd. (Sichuan, China). Phosphatebuffered saline (PBS) was purchased from Shanghai Suobao Biotechnology Co., Ltd. (Shanghai, China). 6gingerol (purity $\geq 98 \%$ ) was purchased from Aladdin (Shanghai, China). 8-gingerol (purity $\geq 98 \%$ ) was purchased from Shanghai Standard Technology Co.,Ltd. (Shanghai, China). Antibodies of anti-IkB $\alpha$ (\#9242 L, Cell Signaling, Shanghai, China), anti-Phospho-p65 of the NF-kB (AN371, Beyotime Biotechnology, Shanghai, China), anti-p65 of NF-kB (sc-109, Santa Cruz Biotechnology, Shanghai, China), and anti- $\beta$-actin (sc47,778, Santa Cruz Biotechnology, Shanghai, China) were purchased commercially. Horse radish peroxidase-conjugated secondary antibodies were purchased from Sigma-Aldrich (Shanghai, China). MMLV(H-) Reverse Transcriptase (R021-01), $2 \times$ Taq Plus Master Mix (P211-01) and ChamQ Universal SYBR qPCR Master Mix(Q711-02) were purchased from Santa Cruz Biotechnology (Dallas, TX, USA). 


\section{Ginger preparation}

Fresh and dried ginger (Zingiber officinale Roscoe) were purchased from Guangdong Provincial Hospital of Traditional Chinese Medicine. For animal studies, air-dried dried ginger rhizomes samples were ground into 200mesh powder. Fine powder was suspended in distilled water and delivered by oral administration. Fresh ginger was ground with a food processor. The juice was then collected after removal of cellular debris by centrifugation at $5000 \times \mathrm{g}$ for $5 \mathrm{mins}$ at room temperature. The liquid was filtered and used as ginger extract for cell culture studies. The property of the extract was profiled by HPLC analysis.

\section{Animals}

Fifty male Specific pathogen-free (6-8 weeks old, 18$220 \mathrm{~g})$ rats were purchased from Laboratory Animal Center of Guangzhou University of Chinese Medicine (Facility approval\# SYXK2018-0044). All rats were housed in a vivarium with a controlled environment and provided with food and water ad libitum. All experiments were performed in accordance with Guidelines for the Care and Use of Laboratory Animals and relevant regulations formulated by Guangdong Provincial Committee for the Care and Use of Laboratory Animals.

\section{IBS-D rats model construction and treatment}

The rat model of IBS-D was established essentially as we previously described [21]. Model group were treated with intracolonic instillation of $1 \mathrm{~mL} 4 \%$ acetic acid at $8 \mathrm{~cm}$ proximal to the anus for $30 \mathrm{~s}$ after anesthetized using $2 \%(0.04 \mathrm{~mL} / 10 \mathrm{~g})$ pentobarbital (intraperitoneal injection). Then, $1 \mathrm{~mL}$ PBS was instilled to dilute the acetic acid and flush the colon. The control group was handled identically, except that $1 \mathrm{~mL}$ saline was instilled instead of $4 \%$ acetic acid. The rats were left to recover from colitis for 7 days. The model group were then subjected unpredictable chronic stress for 3 weeks, followed by 7 days of rest and $1 \mathrm{~h}$ of acute restraint stress on day 28. Food (but not water) was removed $10 \mathrm{~h}$ prior to the acute restraint stress exposure. The unpredictable chronic stress were as we previously described [22]. Afer model construction, the model group were randomly grouped (10 rats per group) and treated with normal saline, rifaxi$\min (50 \mathrm{mg} / \mathrm{kg})$, ginger $(50 \mathrm{mg} / \mathrm{kg}$ or $100 \mathrm{mg} / \mathrm{kg})$ by gastric gavage. For 6-gingerol, intraperitoneal injection was applied on rats with a dose of $10 \mathrm{mg} / \mathrm{kg}$ or 30 $\mathrm{mg} / \mathrm{kg}$ (10 rats per group). Before and after 7 days treatment, all rats were subjected to behavioral evaluation and then the rats were sacrificed by cervical dislocation to obtain the distal colon for future experiments.
Defecation frequency and fecal water content (FWC)

As we previously described [23], the rats were placed in metabolic cages for $24 \mathrm{~h}$ with free access to rodent chow and water. Defecation frequency and FWC were used to estimate the colonic motility and sensibility. The defecation frequency was examined by counting the number of fecal pellets for a period of $1 \mathrm{~h}$. The stool was weighed $(\mathrm{m} 0)$ after collection, and the stool was then weighed again $(\mathrm{m} 1)$ after it was dried in the oven. FWC was calculated as $(\mathrm{m} 0-\mathrm{m} 1) / \mathrm{m} 0$.

\section{Abdominal withdrawal reflex (AWR) scores}

As we previously described [23], AWR scores were used to quantify the colorectal distension (CRD). Simply, the rats were placed in small a cubicle and adapt for $15 \mathrm{~min}$. Then a balloon dilator ( $2 \mathrm{~mm}$ in diameter) was vaselinecoated and inserted into the descending colon which located at $8 \mathrm{~cm}$ proximal to the anus, then water was injected to the balloon, leading to CRD. The AWR score was measured by two independent observers using a double-blind method based on the following criteria: 0 points: no behavioral response to CRD; 1 point: simple head movement, then no movement; 2 points: abdominal muscle contraction; 3 points: abdominal rise; and 4 points: back arch and pelvis ascending. When the AWR score was 3 , the amount of injected water calculated as the degree of CRD. The experiment was repeated in triplicate to obtain an average value.

\section{Pathology analysis}

After the rats were sacrificed, the distal colon was harvested. For pathological analysis, the colon tissues were excised, weighed, and fixed in a tissue fixative buffer, or snap frozen in liquid nitrogen for RNA isolation. Fixed tissues were dehydrated with $4 \%$ paraformaldehyde and then embedded in melted paraffin. The colon tissues were then tailored into $5 \mu \mathrm{m}$-thick sections and stained with HE for morphological evaluations. The stained slices were then subsequently observed and photographed with microscope (Olympus BX53) equipped with a camera. The stained slices were then subsequently observed and photographed with microscope (Olympus BX53) equipped with a camera.

RNA isolation, reverse transcription and real-time qPCR

Colon tissues or cultured cells were used to extract total RNA using TRIzol reagent. The RNA concentrations were determined using NanoDrop 2000. $1 \mathrm{mg}$ RNA was reverse transcribed with $\mathrm{M}-\mathrm{MLV}(\mathrm{H}-)$ Reverse Transcriptase. Real-time PCR was performed on ABI 7300 Real Time PCR System (Applied Biosystems) using ChamQ Universal SYBR qPCR Master Mix. The data were analyzed using the $2^{-\Delta \Delta C t}$ method to obtain relative abundance (Schmittgen\&Livak, 2008). The GAPDH Ct level 
was used as an internal control for normalization. The primer sequences for each gene are listed in Table 1.

\section{Cell cultures and treatment}

As we previously described [21], the distal colons obtained from the model group were cut into small pieces, washed and digested with $0.1 \%$ collagenase I and hyaluronidase for 25 mins at $37^{\circ} \mathrm{C}$ to separate colonic epithelial cell (CECs). After digestion, the supernatant was transferred into a new tube and dulbecco's modified eagle medium (DMEM) was added. After centrifuging 3 times, cells were cultured in DMEM solution containing $100 \mathrm{~mL} / \mathrm{L}$ fetal bovine serum in a $\mathrm{CO}_{2}$ incubator with a saturated humidity at $37^{\circ} \mathrm{C}$. Fibroblasts were removed using phase difference digestion and adherence. When $80-90 \%$ of the cells were adherent to culture plates, 10 , $50,100,200 \mu \mathrm{g} / \mathrm{mL}$ ginger extract and 1, 5, 10, $50 \mu \mathrm{M} 6$ gingerol were used to intervene CECs.

\section{Western blotting}

As we previously described [21-23], total cell lysates were prepared using lysis buffer with 1\% NP-40 and SDS-PAGE protein sample loading buffer supplemented with a cocktail of protease inhibitors. Proteins were separated by SDS-PAGE and transferred to polyvinylidene fluoride membranes, then incubated with their specific antibodies at $4{ }^{\circ} \mathrm{C}$ overnight. $\beta$-actin was used as a loading control.

\section{HPLC analysis}

The component of the ginger was profiled by HPLC analysis. The ginger powder and ginger extract were soaked in methanol for $48 \mathrm{~h}$ and followed by filtration to obtain ginger methanolic extract. Chromatographic separation of the ginger compounds was performed using HPLC (Alliance 2695, Waters, USA) on an Ultimate XB-C18 column $(250 \times 4.6 \mathrm{~mm}, 5 \mu \mathrm{m}$, Welch, China). The mobile phase was composed of $0.1 \%$ phosphoric acid in water $(\mathrm{v} / \mathrm{v}, \mathrm{A})$ and acetonitrile (B), and a gradient elution program with increasing percentage of solvent $B$ was used. The flow rate was $1.0 \mathrm{~mL} / \mathrm{min}$ with the UV detector set at $282 \mathrm{~nm}$. Commercially purchased 6-gingerol and 8gingerol were used as standards.

\section{Statistical analysis}

All measurement data conform to the normal distribution and were expressed as mean \pm SEM. All collected data were analyzed using SPSS 21.0 (SPSS Inc., Chicago, IL, USA) and graphic display using GraphPad Prism 6 software (GraphPad, USA). Paired t-test analysis was used for comparison before and after treatment within the group. Statistical differences between groups were assessed by the one-way analysis of variance (ANOVA) in multiple comparisons. $P$ value of 0.05 or less was considered as significant.

\section{Results}

\section{Ginger ameliorated clinical symptoms in IBS-D rats}

Defecation frequency and FWC were significantly increased, while CRD was significantly decreased in IBS-D rats when compared to the blank group. After medication, the defecation frequency and FWC were significantly decreased when compared to the IBS-D model group $(P<0.05)$, while with no difference between rifaximin and ginger (50 or $100 \mathrm{mg} / \mathrm{kg}$ ) group (Fig. 1a \& b). The CRD in ginger (50 or $100 \mathrm{mg} / \mathrm{kg}$ ) group and rifaximin group were remarkably increased when compared to the IBS-D model group $(P<0.05)$, and no difference between rifaximin and ginger (50 or $100 \mathrm{mg} / \mathrm{kg}$ ) group (Fig. 1c). These results strongly supported that the successful establishment of IBS-D rat model and ginger ameliorated clinical symptoms in IBS-D rats significantly.

\section{Ginger reduced colon pathological edema and proinflammatory gene expression}

We performed histological staining to examine whether ginger had an effect on colonic microinflammation. The intestinal mucosal barrier in the normal rats (blank group) showed integrity mediated by CECs, where as CECs in the IBS-D rats displayed significant edema. As shown in Fig. 2A, the colon tissues in IBS-D model rats had obvious alterations in intestinal mucosa epithelium, including impaired intestinal mucosal integrity and the influx of inflammatory cells were increased. Histological analysis of the rat colonic tissues revealed a significant reduction in granulocytes and CECs edema after the

Table 1 Primers used for gene induction studies

\begin{tabular}{llll}
\hline Gene name & Accession number & Primer pairs (forward and reverse) & Product size (bp) \\
\hline TNF-a & NM_013693 & F: 5'-CTGAACTTCGGGGTGATCGG & 122 \\
IL-6 & R: 5'-GGCTTGTCACTCGAATTTGAGA & 131 \\
iNOS & F: 5'- CTGCAAGAGACTCCATCCAG & 249 \\
& R: 5'- AGTGGTATAGACAGGTCTGTTGG & \\
GAPDH & F: 5'-CAGATCGAGCCCTGGAAGAC & 95 \\
& R: 5'- CTGGTCCATGCAGACAACCT & \\
\hline
\end{tabular}



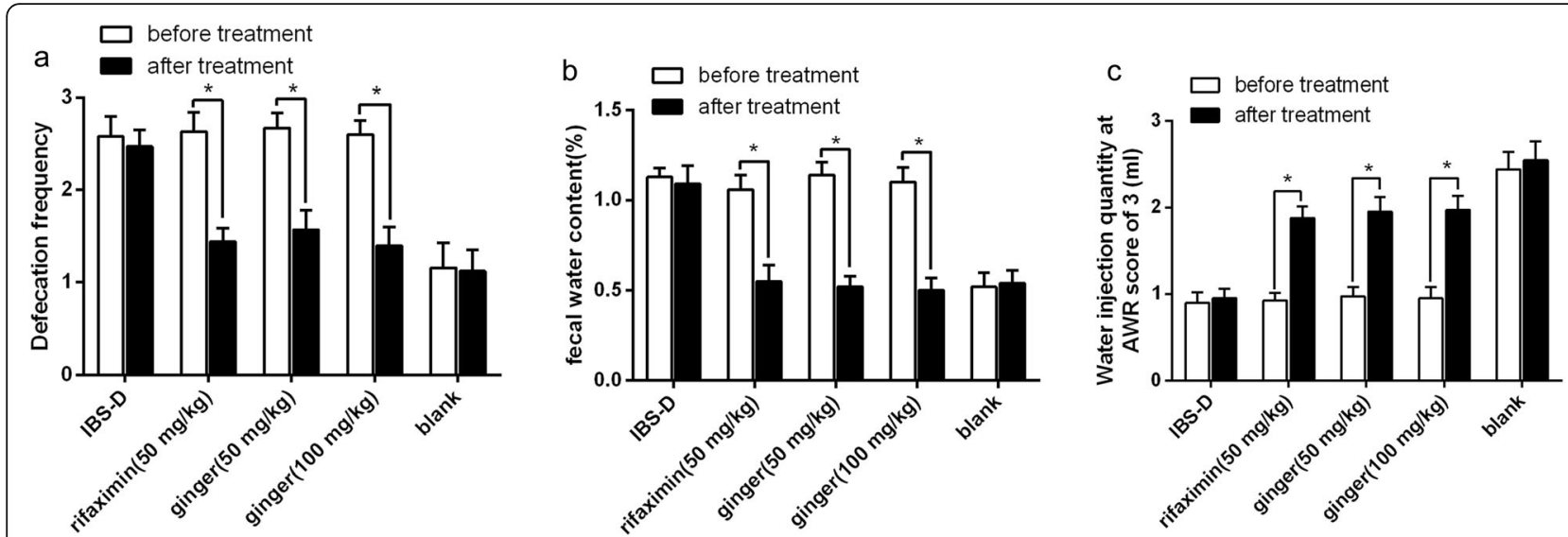

Fig. 1 Effects of ginger on defecation frequency, FWC and CRD in IBS-D rats. a. Ginger treatment decreased defecation frequency in IBS-D rats. b. Ginger treatment decreased FWC in IBS-D rats. c. Ginger treatment increased CRD in IBS-D rats. ${ }^{*} P<0.05$, when compared to before treatment

administration of ginger (50 or $100 \mathrm{mg} / \mathrm{kg}$ ) and rifaximin group, compared to the IBS-D model group and blank group. There was increased monocytic cell infiltrates in IBS-D rats. The intestinal mucosal barrier integrity of mucosal layers were were repaired after oral administration of ginger (50 or $100 \mathrm{mg} / \mathrm{kg}$ ) and rifaximin, and there was no significant difference between ginger (50 or 100 $\mathrm{mg} / \mathrm{kg}$ ) and rifaximin groups. Similar to those in rifaximin-treated group, the structure of the intestinal mucosa epithelium from ginger (50 or $100 \mathrm{mg} / \mathrm{kg}$ ) treated groups remained relatively intact as the blank group. This result strongly suggested that ginger could significantly repair intestinal mucosal integrity and promote histological recovery in IBS-D rats.

The inhibition on inflammation was confirmed by reduced expression of proinflammatory genes in the colon tissues. As shown in Fig. 2B, compared with the IBS-D group, the expression of TNF- $\alpha$, IL- 6 and iNOS in the ginger group was significantly inhibited. In addition, the ginger group at a dose of $100 \mathrm{mg} / \mathrm{kg}$ had better

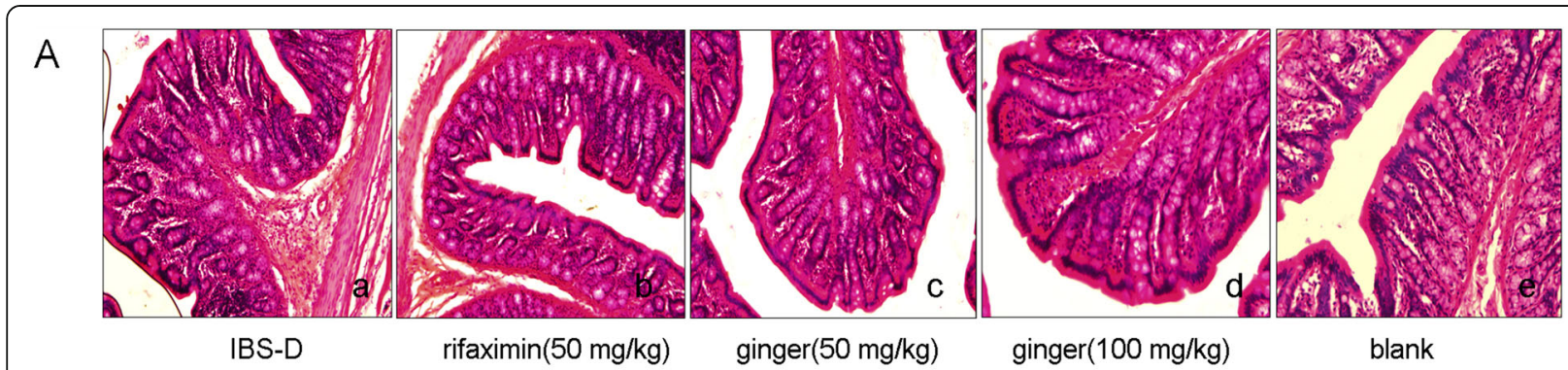

B
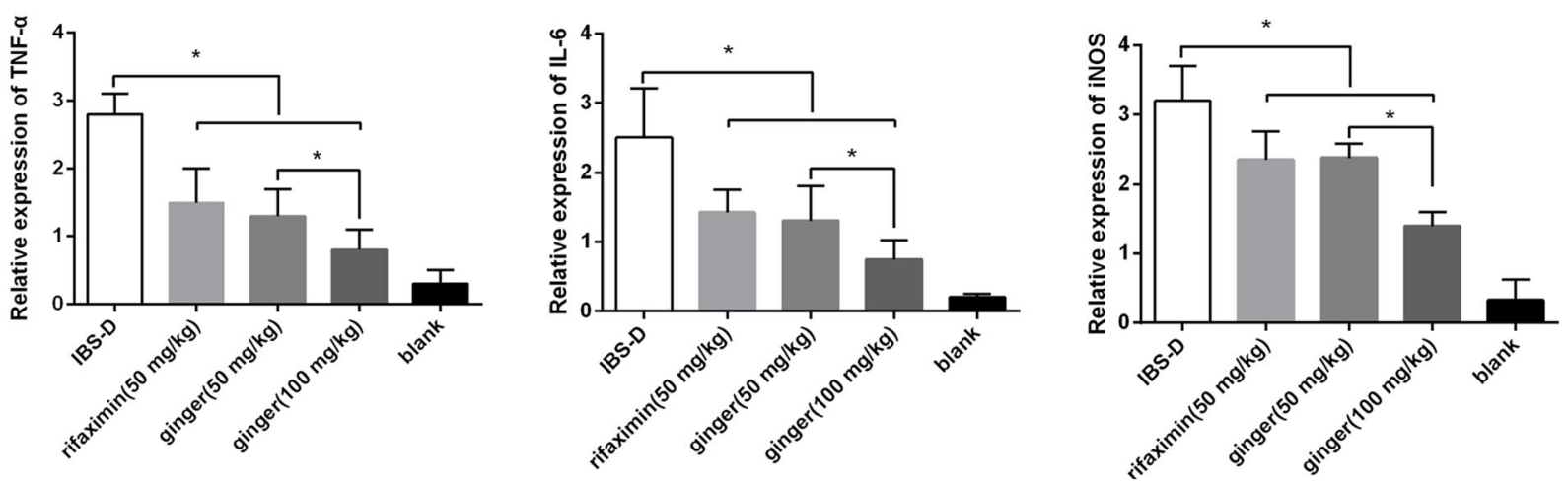

Fig. 2 Ginger treatment reduced pathological edema and proinflammatory gene expression. A. HE stained colonic epithelial tissues. a. IBS-D model group, b. rifaximin (50 mg/kg, po), c. ginger at $50 \mathrm{mg} / \mathrm{kg}$ (po), d. ginger at $100 \mathrm{mg} / \mathrm{kg}$ (po), e. blank group (normal rats). B. Ginger treatment reduced proinflammatory gene expression in the colonic epithelial tissues, detected by RT-qPCR. ${ }^{\#} P<0.05$ indicates significant differences compared to the IBS-D model group. ${ }^{*} P<0.05$ indicates significant differences between ginger groups (50 and $100 \mathrm{mg} / \mathrm{kg}$ ) 

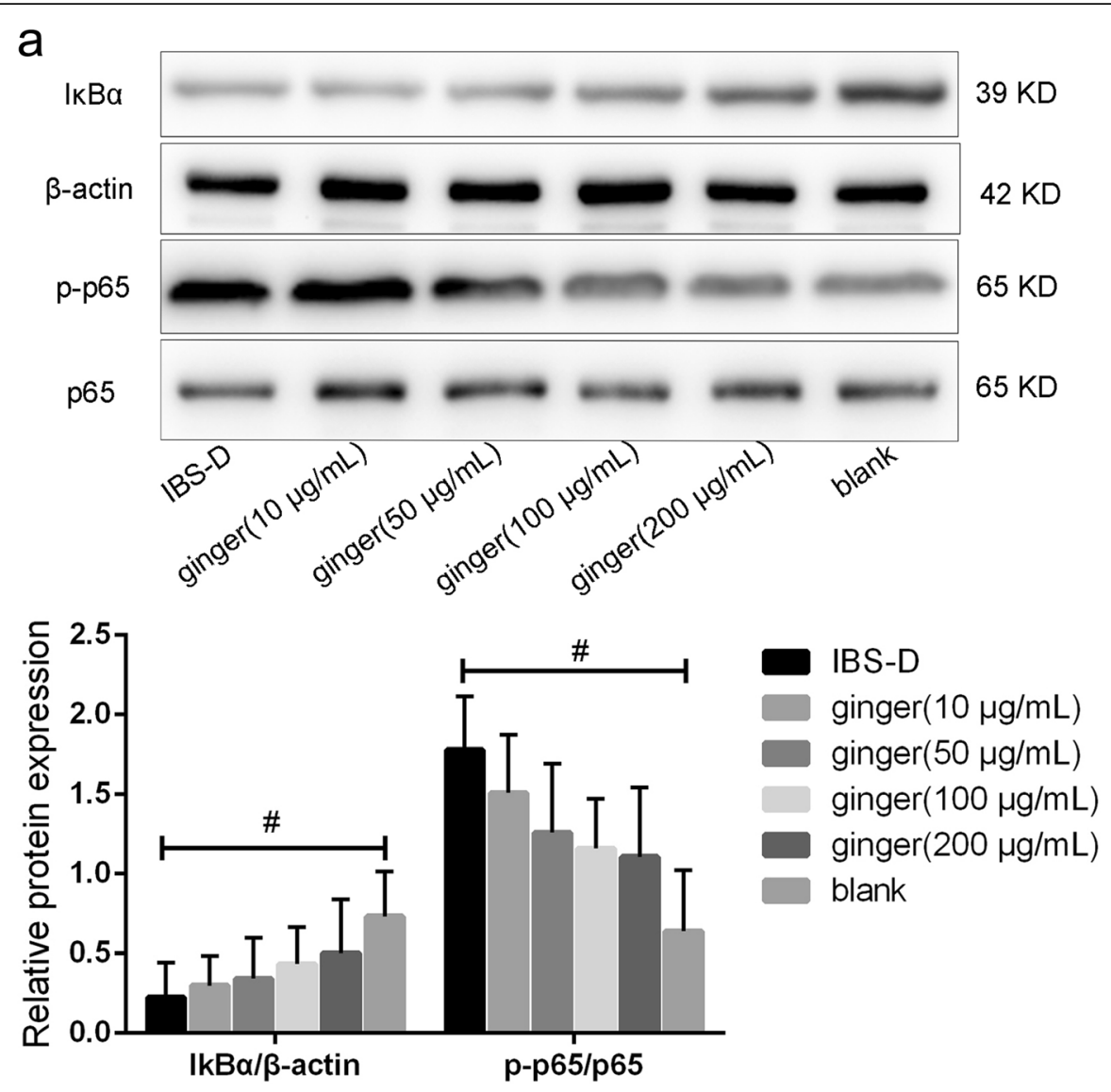

b
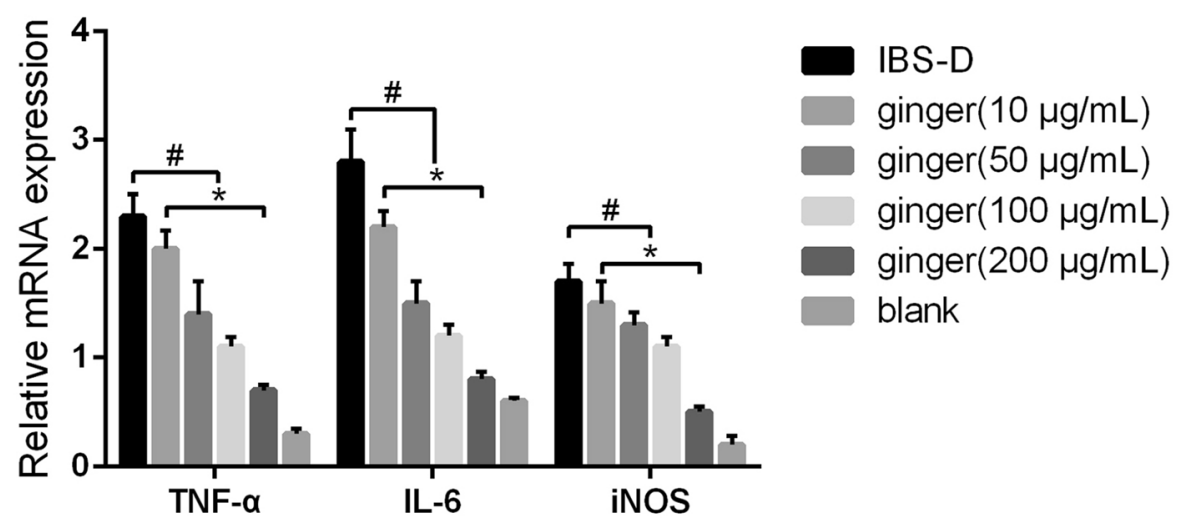

Fig. 3 Ginger extract inhibited NF-KB activation and proinflammatory gene expression in IBS-D rats CECS. a. Western blot showed ginger extract inhibited NF-KB activation in IBS-D rats CECs (up panel). The relative density of IKBa/ $\beta$-actin, p-p65/p65 were also showed (down panel). b. RTqPCR showed ginger extract inhibited proinflammatory gene expression in IBS-D rats CECS. ${ }^{\#} P<0.05$ indicates significant differences between IBSD rats model group and ginger groups $(10,50,100$ and $200 \mu \mathrm{g} / \mathrm{mL}) .{ }^{*} P<0.05$ indicates significant differences between ginger groups $(10,50,100$ and $200 \mu \mathrm{g} / \mathrm{mL})$

inflammation inhibition than the ginger group at a dose of $50 \mathrm{mg} / \mathrm{kg}$. These results indicate that ginger can inhibit the proinflammatory response, which may be related to suppression of intestinal hypersensitivity in IBS-D.
Ginger extract inhibited NF-KB activation and proinflammatory gene expression in IBS-D rats CECs

To further explore the relationship between the relief effect of ginger on IBS-D and proinflammatory response, we focused on the activation pathway of 


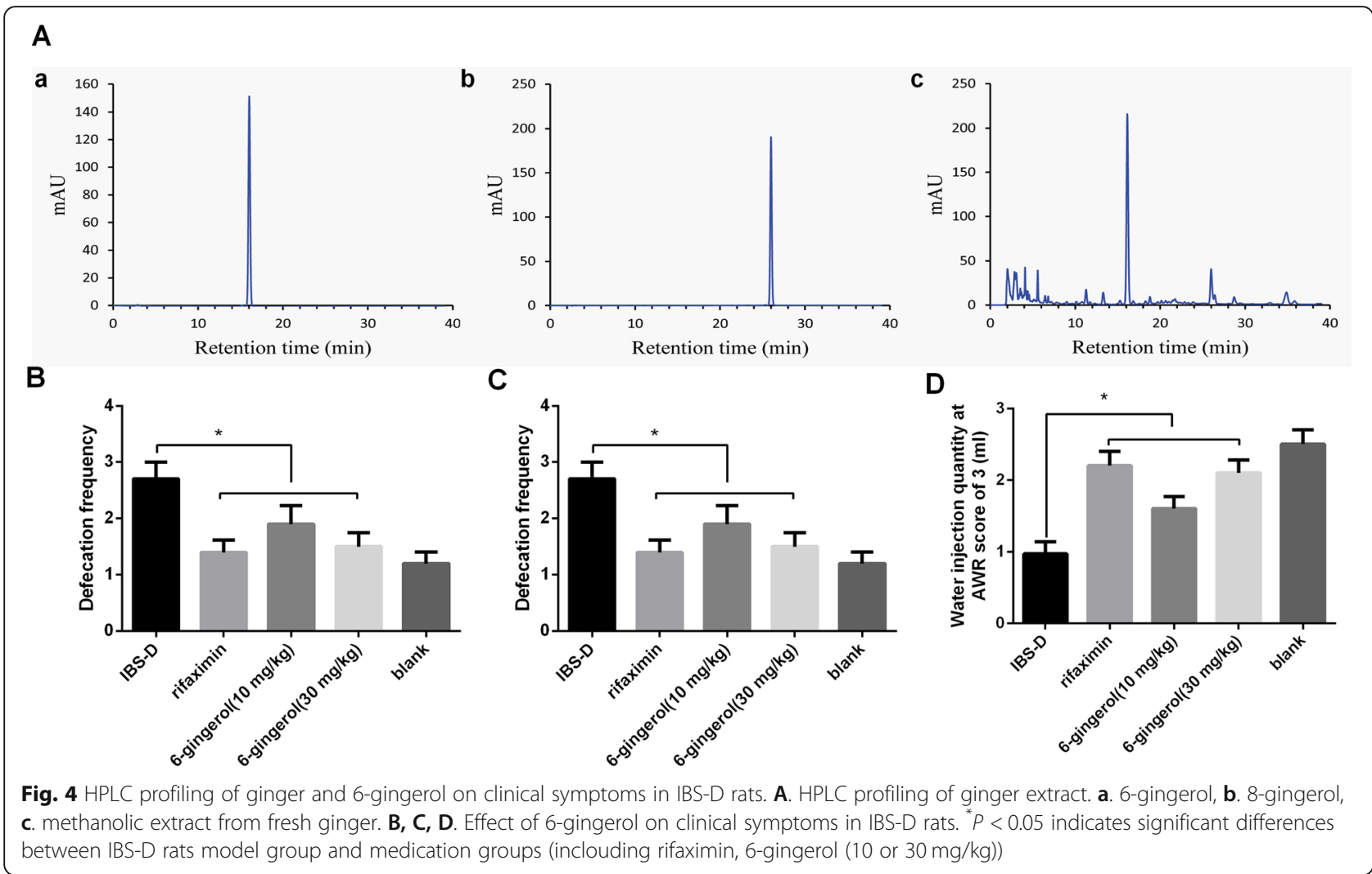

proinflammatory. In this regard, we used IBS-D rats CECs to determine ginger extract wether against NF$\kappa B$ pathway activation. Detected by western blot, ginger inhibited the degradation of $\mathrm{I} \kappa \mathrm{B} \alpha$ and excessive phosphorylation of $\mathrm{p} 65$, a functional subunit of NF$\kappa B$ complex (Fig. 3a, up panel). The relative density of IкB $\alpha / \beta$-actin, p-p65/p65 were also showed (Fig. 3a, down panel). This inhibitory effect was also confirmed at mRNA levels in IBS-D rats CECs. As shown in Fig. $3 \mathrm{~b}$ the CECs from the IBS-D model group significantly increased TNF- $\alpha$, IL- 6 and iNOS mRNA production. Treatment with ginger extract dosedependently suppressed TNF- $\alpha$, IL- 6 and iNOS gene expression in CECs. These results indicated that ginger extract suppressed proinflammatory response by inhibition of NF- $\mathrm{kB}$ pathway activation.

\section{HPLC profiling of ginger and effect of 6-gingerol on clinical symptoms in IBS-D rats}

There are more than 400 different compounds in the ginger extract, with 6-gingerol and 8-gingerol as the major non-volatile, pungent and bioactive principles. We performed an HPLC analysis to identify the presence of gingerols in the extract using commercial 6and 8-gingerol as standards. As shown in Fig. 4A, 6gingerol was among the major component in ginger extract. We then investigated whether 6-gingerol was an effective compound for ginger anti-intestinal hypersensitivity activity using behavioral evaluation. As shown in Fig. 4B $\& \mathrm{C}$, the defecation frequency and FWC were significantly decreased when compared to the IBS-D model group $(P<$ $0.05)$, while with no difference between rifaximin and 6gingerol $(30 \mathrm{mg} / \mathrm{kg})$ group. The CRD in 6-gingerol (10 or $30 \mathrm{mg} / \mathrm{kg}$ ) group and rifaximin group were remarkably increased when compared to the IBS-D model group $(P<0.05)$, and no difference between rifaximin and 6 -gingerol $(30 \mathrm{mg} / \mathrm{kg})$ group (Fig. $4 \mathrm{D})$. This result indicated that 6-gingerol was the effective compound for ginger anti-intestinal hypersensitivity activity in IBS-D rats.

\section{Effect of 6-gingerol on the NF-KB pathway activation and} proinflammatory gene expression in IBS-D rats CECs Further we studied the effect of 6-gingerol on the NF- $k B$ pathway activation and proinflammatory gene expression in IBS-D rats CECs. Detected by western blot, 6-gingerol dose-dependently blocked IкB $\alpha$ degradation as well as p65 phosphorylation in IBS-D rats CECs, which indicated that 6-gingerol showed potent inhibitory activity against IBS-D rats CECs NF-kB pathway activation (Fig. 5a, up panel). The relative density of IкB $\alpha / \beta$-actin, p-p65/p65 were also showed (Fig. 5a, down panel). Similarly, the 6-gingerol also dose-dependently blocked TNF- $\alpha$, IL- 6 , iNOS experssion in IBS-D rats CECs, determined by RT-qPCR (Fig. 5b). 

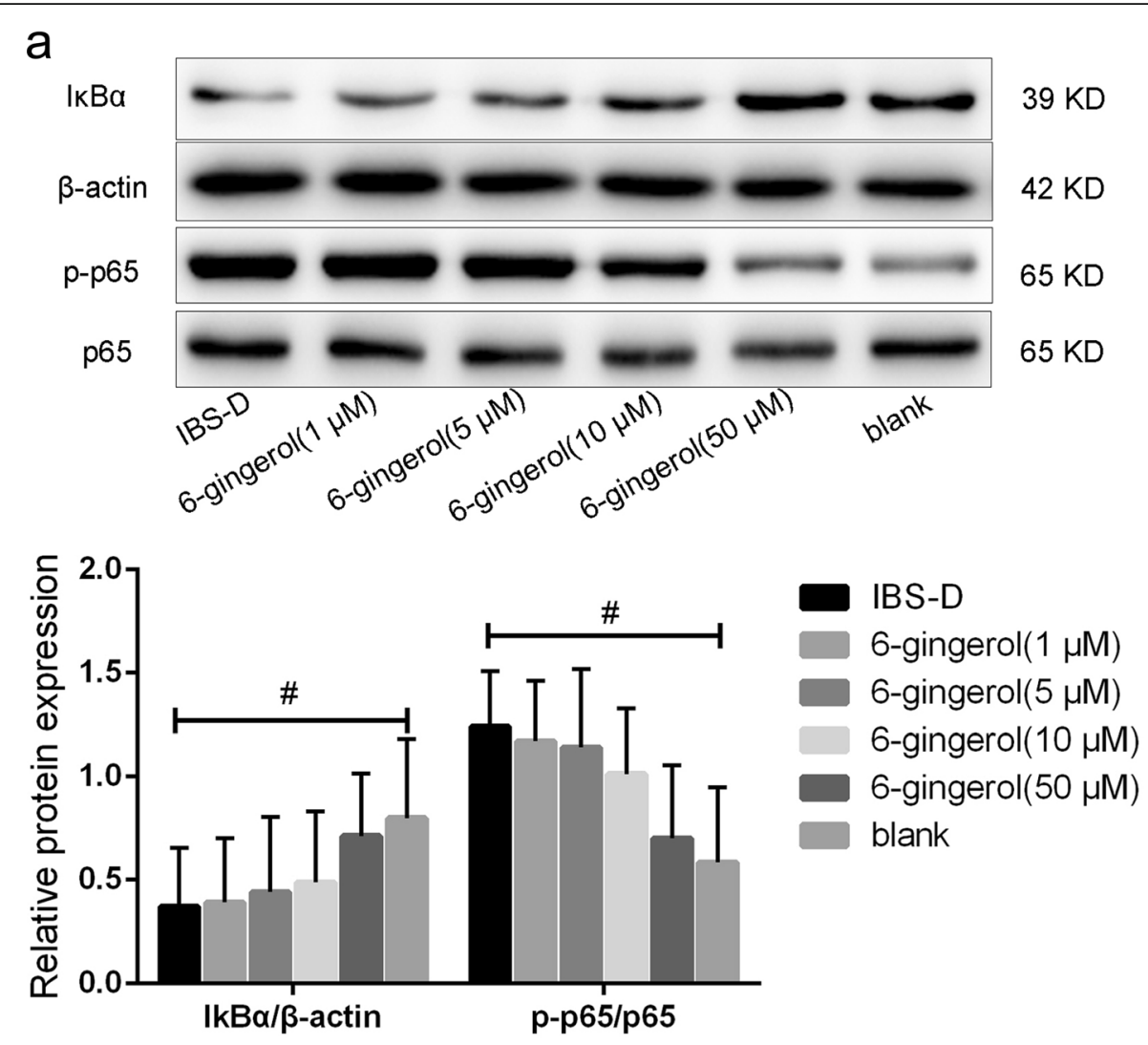

b

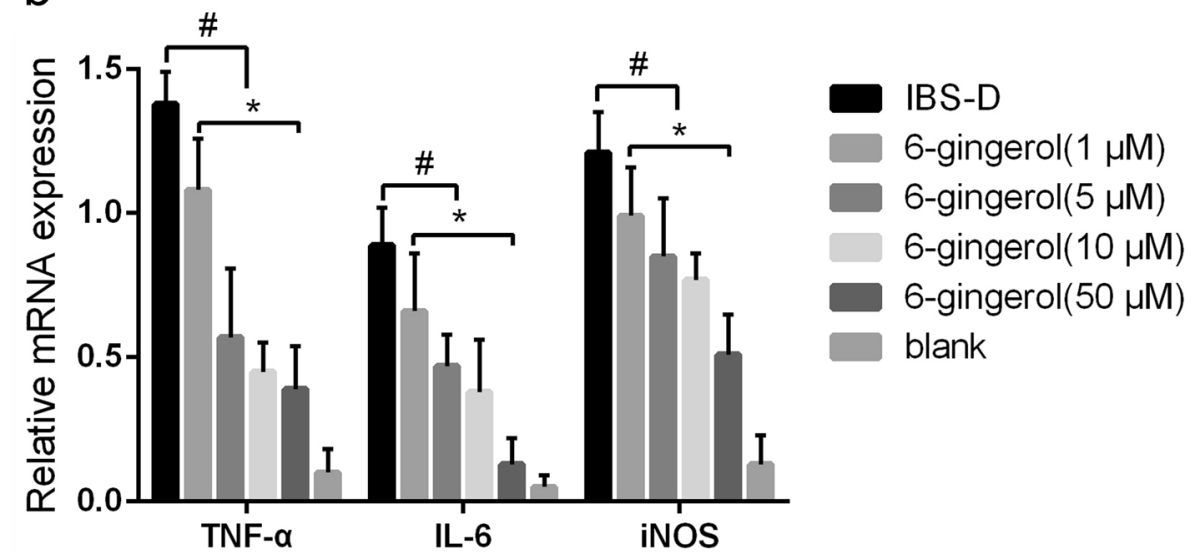

Fig. 5 Effect of 6-ginger on NF-KB pathway activation and proinflammatory gene expression in IBS-D rats CECs. a. Western blot showed effect of 6gingerol on NF-KB activation in IBS-D rats CECS (up panel). The relative density of $\mid k B a / \beta$-actin, p-p65/p65 were also showed (down panel). b. RT-qPCR showed effect of 6-gingerol on proinflammatory gene expression in IBS-D rats CECS. ${ }^{\#} P<0.05$ indicates significant differences between IBS-D rats model group and 6-gingerol groups $(1,5,10$ and $50 \mu \mathrm{M}) .{ }^{*} P<0.05$ indicates significant differences between 6-gingerol groups $(1,5,10$ and $50 \mu \mathrm{M})$

This result demonstrated that 6-gingerol, the major component ginger, had strong anti-intestinal hypersensitivity activity and anti-inflammation activity.

\section{Discussion}

IBS is a clinical syndrome characterized by chronic discomfort or pain in abdomen caused by disturbed bowel habit, which can be elaborately categorized into four subtypes [24]. IBS-D is generally reported as the most common subtype $(28-46 \%)$ of all IBS [2]. IBS-D is a multifactorial disease and many studies have demonstrated that visceral hypersensitivity [25], gut barrier disfunction [26], aberrant microbiota-brain-gut interaction [27] and intestinal motility abnormality [28] are the 
pivotal participators in the pathogenesis. Conventional medications for IBS-D, such as antidiarrheal, antispasmodic,antibiotics and probiotics, often have limited effects and repeated treatment brings about tremendous socio-economic pressure [29]. Therefore, more effective approaches or agents for the treatment of IBS-D are needed. And the therapeutic focus has naturally shifted to traditional medicine.

As a traditional medicine used for thousands of years, ginger has shown remarkable efficacy in inflammatory diseases. In Micromedex, ginger is classified as a broad spectrum antiemetic, and is effective in treating nausea and vomiting associated with pregnancy and surgery [30, 31]. Ginger is also one of the most commonly used herbal medicines by IBS patients, however, the relevant mechanism is not yet clear. The underlying mechanism for reducing abdominal pain involves the production of PGE2 and a pro-inflammatory response [32]. In this study, we found that ginger can improve intestinal hypersensitivity in the IBS-D rat model, and can relieve the symptoms of IBS-D rats diarrhea and abdominal pain, and accompanied by a reduction in proinflammatory response. These results indicate that the therapeutic effect of ginger on IBS-D may be related to its antiinflammatory activity, but the specific treatment mechanism needs to be explored.

The NF- $\mathrm{kB}$ pathway plays a central role in regulating immune and inflammatory processes and thus becomes the target for developing new therapies of inflammatory diseases [33]. Transcriptional control of the vast majority of genes involved in inflammation requires NF- $\mathrm{KB}$ activation [34]. Prior to the activation, NF- $\mathrm{KB}$ is tethered by association with $\mathrm{I} \kappa \mathrm{B} \alpha$, an inhibitory protein that keeps NF- $\mathrm{kB}$ in an inactive state in the cytoplasm [35]. Various stimuli such as proinflammatory factors induces $I \kappa B \alpha$ degradation, which leads to the release of NF-kB. The liberated NF- $\mathrm{KB}$ translocates to the nucleus to regulate transcription of certain genes [36]. We showed that ginger extract as well as 6-gingerol, the most abundant and

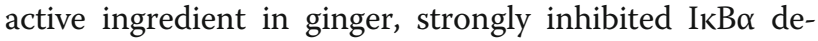
generation and p65 phosphorylation. Therefore, it can be considered that ginger extract and 6-gingerol play an important role in anti-inflammatory in the treatment of IBS-D. Administration of 6-gingerol also improved the clinical symptoms of IBS-D rats.

\section{Conclusion}

Our research shows that ginger treatment can significantly reduce the frequency of defecation and colonic edema in IBS-D rats, and can inhibit the expression of pro-inflammatory factors in IBS-D rats. 6-gingerol has a potential inhibitory effect of pro-inflammatory factors release in CEC of IBS-D rats. Both ginger and 6-gingerol can reduce the intestinal allergies of IBS-D by inhibiting the pro-inflammatory response which involved in NF- $\mathrm{kB}$ signaling pathway. These findings provide evidence that ginger and 6-gingerol are potential protective agents for IBS-D intestinal hypersensitivity. It is necessary to further study the intestinal barrier protective function of ginger and 6-gingerol.

\section{Supplementary information}

Supplementary information accompanies this paper at https://doi.org/10. 1186/s12906-020-03059-3.

\section{Additional file 1.}

Additional file 2

\section{Abbreviations}

IBS: Irritable bowel syndrome; IBS-D: Diarrhea-predominant irritable bowel syndrome; PGE2: Prostaglandin E2; COX-1,COX-2: cyclooxygenase-1 and-2; HE: Hematoxylin-Eosin; FWC: Fecal water content; CRD: Colorectal distension; CECs: Colonic epithelial cell; DMEM: Dulbecco's modified eagle medium

\section{Acknowledgements}

Not applicable.

\section{Authors' contributions}

All authors contributed to this study. QKH and FBL conceived and designed the experiments; CRZ, YQH and PWL performed the experiments; $\mathrm{XLC}$ contributed to statistical analysis of the data; and CRZ and YQH wrote the manuscript. All authors have read and approved the manuscript.

\section{Funding}

This study was supported by the National Natural Science Foundation (No.81804047) (design of the study), Hong Kong scholar program (XJ2018059) (interpretation of data), Innovative Research Team Project of "Innovative Strong Institute", the First Affiliated Hospital of Guangzhou University of Traditional Chinese Medicine (2017TD05) (collection of data).

Availability of data and materials

The data used to support the findings of this study are available from the corresponding author upon request.

Ethics approval and consent to participate

Fifty male Specific pathogen-free (6-8 weeks old, 18-220 g) rats were purchased from the Experimental Animal Center of Guangzhou University of Chinese Medicine (SCXK 2018-0044). The animal study was approved by the Ethics Committee of Guangzhou University of Chinese Medicine.

\section{Consent for publication}

Not applicable.

Competing interests

The authors declare that they have no competing interests.

\section{Author details}

${ }^{1}$ Graduate School, Guangzhou University of Chinese Medicine, Guangzhou, China. ${ }^{2}$ Department of Orthopedics, The Second Affiliated Hospital of Guangzhou University of Chinese Medicine, Guangzhou, China. ${ }^{3}$ Department of Gastroenterology, The First Affiliated Hospital of Guangzhou University of Chinese Medicine, Guangzhou, Guangdong 510405, P.R. China. ${ }^{4}$ Department of Preventive Medicine and Health Statistics, Guangzhou University of Chinese Medicine, Guangzhou, Guangdong, China. ${ }^{5}$ School of Chinese Medicine, Hong Kong Baptist University, Hong Kong, China. 
Received: 2 July 2020 Accepted: 23 August 2020

Published online: 14 September 2020

\section{References}

1. Donnachie E, Schneider A, Mehring M, Enck P. Incidence of irritable bowel syndrome and chronic fatigue following Gl infection: a population-level study using routinely collected claims data. Gut. 2018;67(6):1078-86.

2. Coeffier M, Dechelotte $P$, Ducrotte $P$. Intestinal permeability in patients with diarrhea-predominant irritable bowel syndrome: is there a place for glutamine supplementation? Gastroenterology. 2015;148(5):1079-80.

3. Gracie DJ, Ford AC. Irritable bowel syndrome-type symptoms are associated with psychological comorbidity, reduced quality of life, and health care use in patients with inflammatory bowel disease. Gastroenterology. 2017;153(1): 324-5.

4. Sun J, Wu X, Meng Y, Cheng J, Ning H, Peng $Y$, et al. Electro-acupuncture decreases 5-HT, CGRP and increases NPY in the brain-gut axis in two rat models of diarrhea-predominant irritable bowel syndrome(D-IBS). BMC Complement Altern Med. 2015;15:340.

5. Lembo A, Pimentel M, Rao SS, Schoenfeld P, Cash B, Weinstock LB, et al. Repeat treatment with Rifaximin is safe and effective in patients with diarrhea-predominant irritable bowel syndrome. Gastroenterology. 2016; 151(6):1113-21.

6. Lembo A, Rao SSC, Heimanson Z, Pimentel M. Abdominal pain response to Rifaximin in patients with irritable bowel syndrome with diarrhea. Clin Transl Gastroenterol. 2020;11(3):e00144.

7. van Tilburg MA, Palsson OS, Levy RL, Feld AD, Turner MJ, Drossman DA, et al. Complementary and alternative medicine use and cost in functional bowel disorders: a six month prospective study in a large HMO. BMC Complement Altern Med. 2008;8:46.

8. Srivastava KC, Mustafa T. Ginger (Zingiber officinale) in rheumatism and musculoskeletal disorders. Med Hypotheses. 1992;39(4):342-8.

9. Altman RD, Marcussen KC. Effects of a ginger extract on knee pain in patients with osteoarthritis. Arthritis Rheum. 2001;44(11):2531-8.

10. Park M, Bae J, Lee DS. Antibacterial activity of [10]-gingerol and [12] gingerol isolated from ginger rhizome against periodontal bacteria. Phytotherapy research : PTR. 2008;22(11):1446-9.

11. Young HY, Luo YL, Cheng HY, Hsieh WC, Liao JC, Peng WH. Analgesic and anti-inflammatory activities of [6]-gingerol. J Ethnopharmacol. 2005;96(1-2): 207-10.

12. Tian L, Qian W, Qian Q, Zhang W, Cai X. Gingerol inhibits cisplatin-induced acute and delayed emesis in rats and minks by regulating the central and peripheral 5-HT, SP, and DA systems. J Nat Med. 2020;74(2):353-70.

13. Borrelli F, Capasso R, Aviello G, Pittler MH, Izzo AA. Effectiveness and safety of ginger in the treatment of pregnancy-induced nausea and vomiting. Obstet Gynecol. 2005;105(4):849-56

14. Chaiyakunapruk N, Kitikannakorn N, Nathisuwan S, Leeprakobboon K Leelasettagool $C$. The efficacy of ginger for the prevention of postoperative nausea and vomiting: a meta-analysis. Am J Obstet Gynecol. 2006;194(1):95-9.

15. Ghayur MN, Gilani AH. Pharmacological basis for the medicinal use of ginger in gastrointestinal disorders. Dig Dis Sci. 2005;50(10):1889-97.

16. Terry R, Posadzki P, Watson LK, Ernst E. The use of ginger (Zingiber officinale) for the treatment of pain: a systematic review of clinical trials. Pain Med. 2011;12(12):1808-18.

17. Singh AK, Zajdel J, Mirrasekhian E, Almoosawi N, Frisch I, Klawonn AM, et al. Prostaglandin-mediated inhibition of serotonin signaling controls the affective component of inflammatory pain. J Clin Invest. 2017; 127(4):1370-4.

18. Grabauskas G, Wu X, Gao J, Li JY, Turgeon DK, Owyang C. Prostaglandin E2, produced by mast cells in Colon tissues from patients with irritable bowel syndrome. Gastroenterology: Contributes to Visceral Hypersensitivity in Mice; 2020.

19. Bando T, Fujita S, Nagano N, Yoshikawa S, Yamanishi Y, Minami M, et al. Differential usage of COX-1 and COX-2 in prostaglandin production by mast cells and basophils. Biochem Biophys Rep. 2017;10:82-7.

20. Hwang YH, Kim T, Kim R, Ha H. The Natural Product 6-Gingerol Inhibits Inflammation-Associated Osteoclast Differentiation via Reduction of Prostaglandin E (2) Levels. Int J Mol Sci. 2018;19:7.

21. Hou Q, Huang Y, Zhu S, Li P, Chen X, Hou Z, et al. MiR-144 increases intestinal permeability in IBS-D rats by targeting OCLN and ZO1. Cell Physiol Biochem. 2017;44(6):2256-68.
22. Hou Q, Huang Y, Zhang C, Zhu S, Li P, Chen X, et al. MicroRNA-200a targets cannabinoid receptor 1 and serotonin transporter to increase visceral Hyperalgesia in diarrhea-predominant irritable bowel syndrome rats. J Neurogastroenterology Motility. 2018;24(4):656-68.

23. Hou Q, Huang Y, Zhu Z, Liao L, Chen X, Han Q, et al. Tong-Xie-Yaofang improves intestinal permeability in diarrhoea-predominant irritable bowel syndrome rats by inhibiting the NF-kappaB and notch signalling pathways. BMC Complement Altern Med. 2019;19(1):337.

24. American GA. Irritable bowel syndrome in adults (IBS-D): clinical decision support tool. Gastroenterology. 2019;157(3):855.

25. Simren M, Tornblom H, Palsson OS, Van Oudenhove L, Whitehead WE, Tack J. Cumulative effects of Psychologic distress, visceral hypersensitivity, and abnormal transit on patient-reported outcomes in irritable bowel syndrome. Gastroenterology. 2019;157(2):391-402 e392.

26. Nozu T, Miyagishi S, Nozu R, Takakusaki K, Okumura T. Losartan improves visceral sensation and gut barrier in a rat model of irritable bowel syndrome. Neurogastroenterol Motil. 2020;32(6):e13819.

27. Ibeakanma C, Ochoa-Cortes F, Miranda-Morales M, McDonald T, Spreadbury I, Cenac N, et al. Brain-gut interactions increase peripheral nociceptive signaling in mice with postinfectious irritable bowel syndrome. Gastroenterology. 2011;141(6):2098-108 e2095.

28. Schmidt T, Pfeiffer A, Kaess H. Abnormal intestinal motility in irritable bowel syndrome. Gastroenterology. 1996;111(5):1400-1.

29. Dai C, Jiang M, Sun MJ. Rifaximin in the treatment of patients with diarrheapredominant irritable bowel syndrome. Gastroenterology. 2017;152(6):1629-30.

30. Tavlan A, Tuncer S, Erol A, Reisli R, Aysolmaz G, Otelcioglu S. Prevention of postoperative nausea and vomiting after thyroidectomy: combined antiemetic treatment with dexamethasone and ginger versus dexamethasone alone. Clin Drug Invest. 2006;26(4):209-14.

31. Blumenthal M. Ginger as an antiemetic during pregnancy. Altern Ther Health Med. 2003;9(1):19-21 author reply 19-21.

32. Kiuchi F, Shibuya M, Sankawa U. Inhibitors of prostaglandin biosynthesis from ginger. Chem Pharmaceutical Bull. 1982;30(2):754-7.

33. Hayden MS, Ghosh S. Shared principles in NF-kappaB signaling. Cell. 2008; 132(3):344-62

34. Kunnumakkara AB, Shabnam B, Girisa S, Harsha C, Banik K, Devi TB, et al. Inflammation, NF-kappaB, and chronic diseases: how are they linked? Crit Rev Immunol. 2020;40(1):1-39.

35. Chen J, Lobb IT, Morin P, Novo SM, Simpson J, Kennerknecht K, et al. Identification of a novel TIF-IA-NF-kappaB nucleolar stress response pathway. Nucleic Acids Res. 2018;46(12):6188-205.

36. Habelhah $\mathrm{H}$. Emerging complexity of protein ubiquitination in the NFkappaB pathway. Genes Cancer. 2010;1(7):735-47.

\section{Publisher's Note}

Springer Nature remains neutral with regard to jurisdictional claims in published maps and institutional affiliations.

Ready to submit your research? Choose BMC and benefit from:

- fast, convenient online submission

- thorough peer review by experienced researchers in your field

- rapid publication on acceptance

- support for research data, including large and complex data types

- gold Open Access which fosters wider collaboration and increased citations

- maximum visibility for your research: over $100 \mathrm{M}$ website views per year

At BMC, research is always in progress.

Learn more biomedcentral.com/submissions 